\title{
Multi-Source Knowledge Bases and Ontologies with Multiple Individual and Social Viewpoints
}

\author{
Matthias Nickles, Ruth Cobos, Gerhard Weiss \\ AI/Cognition Group, Department of Computer Science, Technical University of Munich \\ D-85748 Garching b. Muenchen, Germany, \{nickles,cobos,weissg\}@cs.tum.edu \\ Tina Froehner \\ Research Center Knowledge Management (RCKM) \\ D-50678 Koeln, Germany, tina.froehner@fh-koeln.de
}

\begin{abstract}
In open environments like the Web, and open Multiagent and Peer2Peer systems, consent among the autonomous, self-interested knowledge sources and users very often cannot be established, and the estimation of trustability and truthfulness of knowledge sources may not be possible. Moreover, competing viewpoints and their communicative contexts even provide valuable meta-knowledge about the intentions of the participants and their social relationships. As a foundational approach to semantically heterogeneous knowledge perspectives, we introduce a formal framework for the computational representation and integration of multi-source knowledge, which makes explicit heterogeneous viewpoints, and conflicting opinions and their social contexts, and allows for the rating, generalization and optional fusion of knowledge by social choice.
\end{abstract}

Keywords: Semantic Web, Knowledge and Ontology Aggregation and Ranking, Knowledge Provenance, Ontology Merging

\section{Introduction}

Open environments like the (Semantic) Web, and Multiagent and Peer2Peer systems are characterized by properties like openness, opaqueness of true intentions and true beliefs of the participants (trustability might not be given or known), high dynamics and complexity, and the absence of consented and authoritative knowledge in general (especially using media like discussion forums, Wikis or Blogs, and in regard to topics like politics, culture, and the assessment of products, other opinions and people). These issues have in common that they rise mainly from the $a u$ tonomy of knowledge sources and users, being black- or gray-box actors with more or less opaque goals and heterogeneous world views. The way such autonomous entities exchange information is communication. Information on the web can already be considered as communicative, because it is generated in order to influence its recipients and its intentionality and reliability is often unknown. This is even true if knowledge is exchanged indirectly, tacitly or asynchronously using, e.g., static web sites. Like ordinary communication, web knowledge is also contextualized with other knowledge, and it can be agreed as well as denied by other knowledge on the web. Starting from these observations, we pursue the goal to represent knowledge contributions in regard to their communicative (i.e., social) origin, acceptance and use, not their "degree of being true" or reliable as in most related approaches from multi-party knowledge acquisition and belief revision (e.g., [2, 7]). Thereby, we do not restrict ourselves to a specific formal logic, and do for the purpose of this work also not separate assertive knowledge about individuals and ontological knowledge about concepts.

\section{Semantical Perspectives}

As an approach to the described issues, our setting consists of autonomous, self-interested knowledge sources (KSs) and knowledge users (KUs), being, e.g., documents, web sites, data bases, or peers in a Peer2Peer network. We rely on the assumption that both a knowledge source and a knowledge user (and sets like social groups or communities thereof) can be described exhaustively (for our purposes) by her expected more or less uncertain communication attitudes [8] towards arbitrary pieces of knowledge in terms of agreement and contribution. These expectations are maintained and, if necessary, revised dynamically by an observer, who trails communication processes (e.g., observing web sites or agents). 
"Agreement" is simply the attitude of a KU or KS being allegedly in consent (resp. dissent) with a logical statement, and "contribution" states whether the KS is expected to contribute pro-actively a certain statement or type of statement, which means inter alia that the contribution is adequate from the point of view of the contributor in a certain situation, in terms of, e.g., state of argumentation, communication topic and social norms. For example, during a discourse about a certain theme, a $\mathrm{KS}$ is expected to agree even with off-topic statements if these are true in her opinion (e.g., when being asked), but contributes only on-topic statements pro-actively. Both attitudes can depend heavily from the respective social context the KS/KU is situated in, including the set of addresses of a knowledge contribution $(\mathrm{KC})$ and the state of the communication process when uttering the $\mathrm{KC}$. E.g., since KSs are autonomous, self-interested entities, and thus need not to be honest or sincere, a certain KS can express opinion $a$ facing knowledge user $k u_{1}$ and almost simultaneously assert $\neg a$ facing knowledge user $k u_{2}$, even without changing her true beliefs. We represent both attitudes as logical modalities, namely Agrees and Cont(-ributes).

As we want to reason about uncertain attitudes also, we allow for probabilistic weights $\operatorname{Pr}(\ldots) \in[0 ; 1]$, that is, for probabilistic modalities of the form $\operatorname{Pr}\left(\right.$ Agrees $\left._{i d}(f)\right)$ and $\operatorname{Pr}\left(\operatorname{Cont}_{i d}(f)\right)$. At this, id denotes a knowledge source (in case of Agrees and Cont) or a knowledge user (for Agrees only), and $f$ denotes a logical formula from a language denoted as $F$. For lack of space, we focus on the Agrees modality mainly. Other attitudes, like LacksInformation for KUs are also reasonably, but are not investigated in this paper. Social contexts (besides the resp. knowledge targets) are expressed as parts of probability conditions. Precisely, we denote probabilities which depend from a certain social context as $\operatorname{Pr}\left(\right.$ Agrees $_{k s_{i}}(f) \longleftrightarrow$ context $)$ and $\operatorname{Pr}\left(\right.$ Cont $_{k s_{i}}(f) \longleftrightarrow$ context $)$, with context $=\left(k t_{i}\right.$, socialState $), k t_{i}$ being a (set of) identifiers of knowledge user(-s) (e.g., agents, web bots, web surfers...) the agreement (resp. contribution) is addressed to, and socialState denoting a social situation. The latter can comprise many kinds of circumstances, like process states, or logical conditions, or time intervals of the form timePoint start..timePoint $_{\text {end }}$. For the introductional purpose of this work, we use either logical conditions (conditioning the occurrence of communications, not content truth) or topical state names (e.g., Cont $_{\text {seller }}($ statement $) \longleftrightarrow$ (customer, SellingTalk)). A social state can denote past, present and future situations. In case of past and present states, the respective probability becomes 1 or 0 provided the agreement (contribution) is observable. Observe that the social context might be only a part of the (expected) evidence the main- tainer of the probability has. Previous knowledge about the $\mathrm{KSs} / \mathrm{KUs}$, e.g. obtained from trust networks, might contribute additionally to these probabilities.

We can now model viewpoints and opinions of autonomous entities (KSs and KUs) in a unified way in terms of their respective communication attitudes towards knowledge, which we unite under the term perspective $(\theta)$. The $\theta\left[F^{\prime}\right]^{A}$ we are focusing on are called assertive perspectives.

$$
\begin{aligned}
& \theta\left[F^{\prime}\right]^{A}, \theta\left[F^{\prime}\right]^{C}: \text { Id } \times \text { Context } \times F^{\prime} \rightarrow[0 ; 1] \\
& \theta\left[F^{\prime}\right]: I d \times \text { Context } \times F^{\prime} \rightarrow[0 ; 1] \times[0 ; 1] \\
& \left.\theta\left[F^{\prime}\right]_{i d}^{A}(f \longleftarrow \text { context })=\operatorname{Pr}\left(\text { Agrees }_{i d}(f) \longleftarrow \text { context }\right)\right) \\
& \left.\theta\left[F^{\prime}\right]_{i d}^{C}(f \longleftarrow \text { context })=\operatorname{Pr}\left(\text { Cont }_{i d}(f) \longleftarrow \text { context }\right)\right) \\
& \theta\left[F^{\prime}\right]_{i d}(f \longleftarrow \text { context })= \\
& \left(\theta\left[F^{\prime}\right]_{i d}^{A}(f \longleftarrow \text { context }), \theta\left[F^{\prime}\right]_{i d}^{C}(f \longleftarrow \text { context })\right)
\end{aligned}
$$

At this, $F^{\prime} \subseteq F$ is a set of formulas the perspective is restricted to. $\left[F^{\prime}\right]$ is most times omitted in this paper for simplicity. $i d \in I d$ is an unambiguous identifier within a set $I d$ of identifiers of entities currently present in the respective application, denoting either a KS or/and KU (of course, a KU can be a KS also, and vice versa), or simply identifying the respective perspective (i.e., a perspective need not to be explicitly bound to a KU/KS, but can be self-contained also). Technically, e.g. URIs could be used as identifiers. The context is the situation of the entity $i d$ when he expresses his opinion with the respective probability. $\longleftarrow$ context can be omitted in order to obtain the probability in absence of any contextual information. If, e.g., $\theta_{\text {sellerAgent }}^{A}\left(\right.$ heavy $($ camera $) \longleftrightarrow\left(\right.$ customer $\left.\left._{1}, \ldots\right)\right)=1$ and

$\theta_{\text {seller Agent }}^{A}\left(\neg\right.$ heavy $($ camera $) \longleftrightarrow\left(\right.$ customer $\left.\left._{2}, \ldots\right)\right)=1$, an observer can conclude $\theta_{\text {seller Agent }}^{A}($ heavy $($ camera $))=$ 0.5 if no context is given.

In case the entity $i d$ can be queried (i.e., acts in a collaboratively manner upon questions), or has a publicly visible content (like a web page), $\theta^{A}$ can be derived from $\theta^{C}$ using

$\theta_{i d}^{A}(f \longleftrightarrow$ context $)=$

$\theta_{i d}^{C}(f \longleftarrow($ observer, Queries observer $(f) \cup$ context $))$, with Queries observer $(f)$ denoting a situation in which entity $i d$ is asked iff he agrees with $f$.

Recall in this respect that of course a perspective does not need to reflect the true (mental) belief of the agreeing/contributing entity. It is also important to see that a perspective is only a more or less uncertain model even of the communication stance of such entities, obtained by an observer from past experience and background knowledge. If the observer has no knowledge about the attitude of $i d$ towards a formula $f$, a way to express this is to set $\theta_{i d}(f \longleftarrow$ 
context $)=(0.5,0.5)$, denoting a uniform distribution of such unknown attitudes.

Perspectives show some resemblance to logical views [3] and contexts (as in context logic) (e.g., [6]), and assumably the latter could be modeled as a special case of an assertive perspectives without probabilities and without social contexts (which provide in general no truth conditions for formulas, but pragmatic conditions for their communicative support, usage etc.). We demand that

$$
\begin{gathered}
\left(\theta_{i d}^{A}(f \longleftarrow c)=1 \wedge \theta_{i d}^{A}\left(f \rightarrow f^{\prime} \longleftarrow c\right)=1\right) \\
\rightarrow \theta_{i d}^{A}\left(f^{\prime} \longleftarrow c\right)=1 \\
\theta_{i d}^{A}(f \longleftarrow c) \geq \theta_{i d}^{C}(f \longleftarrow c)
\end{gathered}
$$

and

$$
\left(\theta_{i d}^{A}(f \longleftarrow c)=p\right) \rightarrow\left(\theta_{i d}^{A}(\neg f \longleftarrow c)=1-p\right)
$$

In order to model social perspectives, each of multiple agents, we also allow for sets of KSs and KUs (cf. 3.1 and 3.2 also for the fusion of multiple individual perspectives): $\theta_{\left\{i d_{1}, \ldots, i d_{n}\right\}}^{A}(f \longleftarrow$ context $)=$

$$
\frac{\sum_{i=1}^{n} \theta_{i d_{i}}^{A}(f \longleftarrow \text { context })}{n}
$$

$\theta_{\left\{i d_{1}, \ldots, i d_{n}\right\}}^{C}$ is lifted analogously.

\section{Perspective Generation and Taking}

An observer aiming at modeling knowledge sources or users might faces the situation that only a subset of formulas is given as positive or negative evidence for the respective perspective (e.g., explicitly given as a technical KB, or from a voting process). In this case, the observer needs to generate missing information from the given core set of explicit examples. A set of such examples is given in the form of a so-called quoted knowledge base (QKB):

A quoted knowledge base is a set of formulas annotated with $\mathrm{KS} / \mathrm{KU}$ identifiers, i.e., $\left\{\left(i d_{1}, f_{1}\right), \ldots: i d_{i} \in I d, f_{i} \in\right.$ $F\}$.

The generation of a perspective (without considering its contexts) from such a QKB $q k b$ can be done as follows: $\forall f \in F^{\prime}, \theta\left[F^{\prime}\right]_{i d}^{A}=g e n_{i d}^{A}(q k b)$, with

$$
\begin{array}{r}
g e n_{i d}^{A}(q k b, f)=1 \\
\leftrightarrow \exists\left(i d, f^{\prime}\right) \in q k b: f^{\prime} \vdash f \\
g e n_{i d}^{A}(q k b, f)=0 \\
\leftrightarrow \exists\left(i d, f^{\prime}\right) \in q k b: \neg f^{\prime} \vdash f \\
g e n_{i d}^{A}(q k b, f)=0.5 \\
\leftrightarrow\left(\neg \exists\left(i d, f^{\prime}\right) \in q k b: f^{\prime} \vdash f\right) \\
\wedge\left(\neg \exists\left(i d, f^{\prime}\right) \in q k b: \neg f^{\prime} \vdash f\right)
\end{array}
$$

or alternatively:

$g e n_{i d}^{A}(q k b, f)=\operatorname{semanticDist}\left(f,\left\{f^{\prime}:\left(f^{\prime}, i d\right) \in q k b\right\}\right)$
The KB content is treated as being non-monotonic, since it is used as evidence for a perspective, and not as an exhaustive description of it. semanticDist measures the semantic distance of a formula and other formulas. Being out of the scope of this paper, please refer to [4] for details.

The inverse way (obtaining a QKB or a $\mathrm{KB}$ from perspectives) is covered at the end of this section in the context of socially reified knowledge bases.

We can create a set of possibly heterogeneous perspectives $\left\{\theta_{1}, \ldots, \theta_{n}\right\}$ from a single possibly inconsistent unquoted knowledge bases $k b=\left\{f_{i}: f_{i} \in F\right\}$ using some perspective grouping function. This can be considered as some kind of "reverse engineering" of a multi-source knowledge base. group might take into account the degree of inconsistency and/or the topical distances within the KB. We restrict ourselves here to a simple case where the grouping function creates so-called maximally-consistent subsets of KBs [5]:

$$
\begin{array}{r}
\left\{\theta_{i d 1}^{A}, \ldots, \theta_{i d n}^{A}\right\}= \\
\left\{g_{e n}^{A} A\left(\left\{\left(k s_{1}, i d 1\right), \ldots,\left(k s_{1_{1} m}, i d 1\right)\right\}\right)\right. \\
\ldots g e n_{i d n}^{A}\left(\left\{\left(k s_{n}, i d n\right), \ldots,\left(k s_{n_{n} m}, i d n\right)\right\}\right): \\
\left\{k s_{i}, \ldots, k s_{i_{i} m}\right\} \in \operatorname{con}(k b) \wedge \\
\left.\forall c e \in \operatorname{con}(k b): \neg\left(\left\{k s_{i}, \ldots, k s_{i_{i} m}\right\} \subset c e\right)\right\}
\end{array}
$$

using $\operatorname{con}(k b)=\{k s \subseteq k b: k s \nvdash \perp\}$ (i.e., all consistent subsets in $k b$ ).

A single perspective can be obtained from a possibly inconsistent KB using learning techniques from, e.g., Stochastic Logic Programming, given that the KB consists of ground formulas. The merging of perspectives (cf. below) is also a way to obtain probabilistically consistent perspectives from inconsistent knowledge bases, given that this knowledge base is given indirectly as a set of perspectives.

Grouping can also be done with a QKB instead of a KB in order to retrieve "knowledge communities", i.e., sets of virtual perspectives corresponding to sets of KUs/KSs (resp. their identifiers) assumably sharing certain knowledge and thus forming social groups emergent from communicated opinions. Again, with the simple consistency criterium:

$$
\begin{array}{r}
\left\{\theta_{i d_{1_{1}} \sqcup \ldots \sqcup i d_{1_{1}}}^{A}, \ldots, \theta_{i d_{n_{1}} \sqcup \ldots \sqcup i d_{n_{n} m}}^{A}\right\}= \\
\left\{g_{i_{1_{1}} \sqcup \ldots \sqcup i d_{1_{1} m}}^{A}\left(\left\{\left(k s_{1}, i d_{1_{1}} \sqcup \ldots \sqcup i d_{1_{1} m}\right), \ldots\right\}\right)\right. \\
\ldots g e n_{i d_{n_{1}} \sqcup \ldots \sqcup i d_{n_{n} m}}^{A}\left(\left\{\left(k s_{n}, i d_{n_{1}} \sqcup \ldots \sqcup i d_{n_{n} m}\right), \ldots\right\}\right): \\
\left\{\left(k s_{i}, i d_{i_{1}}\right), \ldots,\left(k s_{i_{i} m}, i d_{i_{i} m}\right)\right\} \in \operatorname{con}^{\prime}(q k b) \wedge \\
\forall c e \in \operatorname{con}^{\prime}(q k b): \neg\left(\left\{k s_{i}, \ldots, k s_{i_{i} m}\right\} \subset \operatorname{dropId}(c e)\right\}(14)
\end{array}
$$$$
\text { using } \operatorname{con}^{\prime}(q k b)=\{q k s \subseteq q k b: \operatorname{dropId}(q k s) \nvdash \perp\}
$$$$
\text { (i.e., all consistent subsets in } q k b \text { ignoring quotation) and }
$$$$
\operatorname{dropId}\left(\left\{\left(f_{1}, i d_{1}\right) \ldots\right\}\right)=\left\{f_{1} \ldots\right\} \text {. }
$$

The $i d_{j_{1}} \sqcup \ldots \sqcup i d_{j_{j} m}$ denote composite "community" identifiers, consisting of multiple identifiers of KS contribut(12) ing consistent sets of formula within the $q k b$. Note that such 
"communities" can overlap. Topical grouping using $\theta^{C}$ instead of $\theta^{A}$ would work analogously again, but would require a grouping function which groups those formulas contributing to the same topic (e.g., the identifiers of contributors of inconsistent formulas like $a$ and $\neg a$, but also fast (digicam 1$)$, slow (digicam 1$)$ and camera(digicam) would be considered to be within the same topical community).

Given a set of multiple perspectives, we can state easily how the elements of a certain knowledge base (including ontologies) are "viewed" (agreed, liked...) from these perspectives. This leads to a socially reified knowledge base (SRKB):

A socially reified knowledge base $\operatorname{srkb}(p)$ over a set of pairs of perspectives and social contexts $p=\left\{\left(i d_{i}, c_{j}\right), \ldots,\left(i d_{n}, c_{n}\right)\right\}$ is defined by

$$
\begin{array}{r}
\operatorname{srkb}(p)= \\
\left\{\left(f_{i},\left\{i d_{1} \longleftarrow c_{1}{ }^{\prime}:^{\prime} \theta_{i d_{1}}\left(f_{i} \longleftarrow c_{1}\right),\right.\right.\right. \\
\left.\left.\left.\ldots, i d_{n} \longleftarrow c_{n}{ }^{\prime}{ }^{\prime} \theta_{i d_{n}}\left(f_{i} \longleftarrow c_{i}\right)\right\}\right): f_{i} \in F\right\}
\end{array}
$$

Example:

$\{($ fast $($ digicam $),\{$ buyer $\longleftrightarrow \quad(\{\ldots\}$, Testing $) \quad:$ $(0.4,0)$, seller $\longleftrightarrow(\{$ buyer $\}$, Selling $):(1,1)\})$, seller $\longleftarrow$ $(\{\ldots\}$, Testing $):(0.3,0)\})\}$. Here, KU buyer is expected to approve that a certain digital camera is fast with probability 0.4 when testing it, but is not expected to utter this opinion pro-actively. KS seller approves that the camera is fast with probability 1 in a selling talk (context Selling), and with probability 0.3 when testing it by himself.

A QKB or SRKB can be obtained via the annotation of an ordinary $\mathrm{KB}$, or (trivially) from a set of perspectives directly. Since a perspective might generate attitudes (Agrees, Contributes) for every possible formula $f$ ( $0.5 \mathrm{de}-$ noting maximal uncertainty), restricting the inclusions using a filter function appears to be reasonable, e.g. filtering out formulas and perspective annotations using a condition like $\varepsilon \leq \theta_{i d}^{A / C}(f) \leq 1-\varepsilon$.

We denote $\mathrm{QKBs} / \mathrm{SRKB}$ s obtained from perspectives as $\operatorname{srkb}\left(\left\{\theta_{i d i}\right\}\right)$ (resp. $\left.q k b\right)$.

An un-quoted KB $k b$ (pset, context) obtained from a set of perspectives pset is defined as $\left\{f: \exists \theta_{i d}^{A} \in\right.$ pset : $\theta_{i d}^{A}\left(f^{\prime} \longleftarrow\right.$ context $\left.)=1 \wedge f^{\prime} \vdash f\right\} \cup\left\{\neg f: \exists \theta_{\text {id }}^{A} \in\right.$ pset : $\theta_{i d}^{A}\left(f^{\prime} \longleftarrow\right.$ context $\left.)=0 \wedge \neg f^{\prime} \vdash \neg f\right\}$. Obviously, such a $\mathrm{KB}$ can be inconsistent.

How a set of perspectives can be retrieved from a QKB has been shown already. Analogously, we define the set of perspectives obtained from a subset $s r k b^{\prime}$ of some socially reified knowledge base to be $\operatorname{pset}\left(s r k b^{\prime}\right)$. This is done basically in the same way as in the QKB case (details omitted for lack of space).

\subsection{Perspective Merging and Generalization}

Perspectives can be merged using social choice techniques. For our purpose, we outline several methods to form a single merged perspective from a set of other perspectives (for lack of space for the merger of pairs of assertive perspectives only). Of course, these approaches can be combined and extended in various ways.

Majority voting Given a set of (assertive) perspectives $\left\{\theta_{i d i}^{A}: i d i \in I d\right\}$ and a formula $f$, for the resulting perspectives the following holds in this case: $\theta_{\text {merger }}^{A}(f \longleftarrow c)=\max \left(\min \left(\mid\left\{\theta_{i d i}^{A}: \theta_{i d i}^{A}(f \longleftarrow\right.\right.\right.$ $\left.\left.c) \geq \gamma\}|-|\left\{\theta_{\text {idi }}^{A}: \theta_{\text {idi }}^{A}(f \longleftarrow c)<\gamma\right\} \mid, 1\right), 0\right)$, with $\gamma$ being some trigger value for approval (e.g., 1).

Social power or reputation In case there is a linear order $i d_{1} \succ i d_{2} \succ \ldots i d_{n}$ or values $\operatorname{rep}\left(i d_{i}\right) \in[0 ; 1]$ given (obtained e.g. from a Social Network), denoting the social power or the reputation (resp. trustability, credibility...) of some $\mathrm{KS}$, it can be used to weight perspectives, for example using

$\theta_{\text {merger }}^{A}(f \longleftarrow c)=\frac{\sum_{i=1}^{n} \theta_{i d_{i}}^{A}(f \longleftarrow c) \cdot r e p\left(i d_{i}\right)}{n}$. In [2], a similar approach has been used in the context of dynamic knowledge bases.

Averaging This is simply achieved with $\theta_{\text {merger }}^{A}=\theta_{\{i d i: i d i \in I d\}}^{A}$. We consider this to be the "purest" method to determine the social support for some assertion, for our purpose even in favor of commonly used "biased" ways like majority voting or the consideration of KS reputation. Our kind of averaging is related to Bayesian Aggregation (e.g., [1]), but aggregates and retrieves, as described in section 2 , the context-depended probabilities of communicative actions supporting formulas instead.

Supporters vs. Opponents Similar to averaging, but under consideration of those perspective only which certainly have a determined opinion about the formula:

$\theta_{\text {merger }}^{A}(f \longleftarrow c)=\frac{\mid \text { supporters }_{f} \mid}{\mid \text { supporters }_{f}|+| \text { opponents }_{f} \mid}$,

with supporters $s_{f}=\left\{\theta_{i d_{i}}^{A}(f \longleftarrow c): \theta_{i d_{i}}^{A}(f \longleftarrow c)=\right.$ $1\}$, opponents $f=\left\{\theta_{i d_{i}}^{A}(f \longleftarrow c): \theta_{i d_{i}}^{A}(f \longleftarrow c)=0\right\}$.

Strictly common ground

$$
\theta_{\text {merger }}^{A}(f \longleftarrow c)=1-\min \left(\mid\left\{\theta_{i d i}^{A}: \theta_{i d i}^{A}(f \longleftarrow c)<\right.\right.
$$
$1\} \mid, 1)$

In addition to the merging of multiple perspectives, generalization is also concerned with the preceding selection of which perspectives to be merged. We will need this in 3.2 to combine perspectives according various criteria in order to reduce the complexity of the possibly very large number of individual perspectives in environments like the internet (e.g., one user of a socially reified KB might be interested in the viewpoint of "the average contributor" only, whereas 
other might want to know the opinions of all single contributors). This is achieved by selecting and subsequently merging subsets of perspectives from a larger set pset. The specific technique proposed here is derived from the simple observation, that a (plain) knowledge base obtained from a single perspective should be consistent, whereas a KB obtained from the union of multiple such $\mathrm{KBs}$ is maximal inconsistent, compared to the union of every subset of such KBs. We use this for a leveled generalization, where each generalization step obtains mergers from mutually "rather consistent" perspectives obtained recursively by the preceding step. The idea thereby is that having multiple consistent perspectives annotating a KB is assumably uninteresting (because these perspectives a likely similar anyways), and thus such perspectives should be merged earlier than rather inconsistent perspectives (surely an assumption that might be not adequate for every application).

$$
\begin{aligned}
& \text { generalize }(\text { pset }, \text { merge }, \text { steps, context })=
\end{aligned}
$$

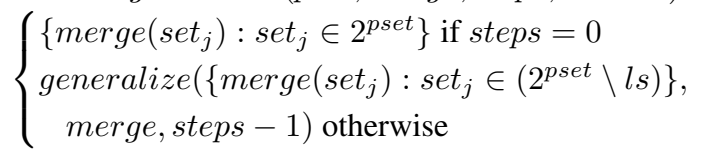

with $l s=s e t_{0}^{\prime}, k b_{0} \preceq k b_{1} \preceq \ldots \preceq k b_{n}$, $k b_{i}=k b\left(\right.$ set $_{i}^{\prime}$, context $),\left\{\right.$ set $\left._{i}^{\prime}\right\}=2^{\text {pset }}$.

At this, merge is a merging function, and $\preceq$ is a inconsistency score ordering [5] (intuitively, $k b_{1}$ is less inconsistent that $k b_{1}$ if $\left.k b_{1} \preceq k b_{2}\right)$. Observe that $l s$ is determined non-deterministically (a score ordering is not unequivocal), so there is a choice of which element of the power set should be dropped at each generalization step.

\subsection{Opinion Bases}

Opinion bases (OB) are a basic kind of Open Knowledge Bases [9], defined upon socially reified knowledge bases, with special features making them more easy to use and to display. Specifically, i) the perspectives used to reify knowledge are obtained from the $\mathrm{KB}$ itself (making the $\mathrm{KB}$ reflective), ii) a number of generalization steps is applied to the $\mathrm{KB}$, and iii) the $\mathrm{KB}$ is presented as a table. Formally, an opinion base is a tuple with

OpBase(srkb, cset, generalize, step, merge, rows),

- $s r k b$ being a subset of a socially reified knowledge base,

- $c$ set being a set of social contexts the creator of the $\mathrm{OB}$ is interested in,

- generalize being a generalization function (cf. previous section),

- step $\in \mathbb{N}_{0}$ being the generalization step of the opinion base,
- merge being a merger function such that $\operatorname{merge}\left(\left\{\theta_{i \operatorname{inn}_{j}}: i \operatorname{iin}_{j} \in I d\right\}\right)=\theta_{i d o u t}$, and

- rows $=\left\{\right.$ rowcluster $\left._{i}\right\}$ being a set of row clusters (the "output" of the OB), each corresponding to a different formula within $s r k b$. Hereby, each rowcluster $_{i}$ is a set $\left\{\left(i d_{j}, c_{k}, \theta_{i d_{j}}\left(f_{l} \hookleftarrow c_{k}\right)\right): i d_{j} \in\right.$ $i d s\left(\right.$ generalize (pset (srkb), merge, step, $\left.\left.c_{k}\right)\right), c_{k} \in$ cset, $f_{l} \in$ formulas $\left.\left.(\operatorname{srkb})\right)\right\}$.

Recall that pset $(s r k b)$ retrieves a set of perspectives from a SRKB. formulas shall obtain the plain KB from a SRKB (i.e., stripped from perspective annotations), and $i d$ shall retrieve the identifiers in a set of perspectives.

Acknowledgements. This work is supported by DFG under contract no. BR609/13-1.

\section{References}

[1] J.A. Benediktsson, K. Arnason. Fusion of Multitype Data. Proc. Workshop on Intelligent Techniques for SpatioTemporal Data Analysis in Environmental Applications, 1999.

[2] J. A. Leite, J. J. Alferes, L. M. Pereira. On the Use of Multi-dimensional Dynamic Logic Programming to Represent Societal Agents Viewpoints. Proc. 10th Portuguese International Conference on Artificial Intelligence (EPIA01), 2001.

[3] J. Ullmann. Information Integration Using Logical Views. Proc. 6th International Conference on Database Theory. Springer, 1997.

[4] J. F. Roddick, K. Hornsby, D. De Vries. A Unifying Semantic Distance Model for Determining the Similarity of Attribute Values. Proc. 26th Australasian Computer Science Conference (ACSC2003), 2003.

[5] A. Hunter, S. Konieczny. Approaches to Measuring Inconsistent Information. Proc. Inconsistency Tolerance 2005, 2005.

[6] A. Farquhar, A. Dappert, R. Fikes, W. Pratt, Integrating Information Sources using Context Logic. Proc. of the AAAI Spring Symposium on Information Gathering from Distributed Heterogeneous Environments, 1995.

[7] A. Dragoni, P. Giorgini. Revisining beliefs received from multiple sources. H. Roth and M. Williams (Eds.): Frontiers in Belief Revision, Kluwer, 431-444, 2001.

[8] M. Nickles, F. Fischer, G. Weiss. Communication Attitudes: A Formal Approach to Ostensible Intentions and Individual and Group Opinions. Proc. 3rd International Workshop on Logic and Communication in Multi-Agent Systems (LCMAS). 2005.

[9] M. Nickles, T. Froehner, G. Weiss. Social Annotation of Semantically Heterogeneous Knowledge. 4th International Workshop on Knowledge Markup and Semantic Annotation (SemAnnot) @ ISWC'04. 2004. 